\title{
Supernovae-generated high-velocity compact clouds
}

\author{
A. Yalinewich ${ }^{1}$ and P. Beniamini ${ }^{2,3}$ \\ ${ }^{1}$ Canadian Institute for Theoretical Astrophysics, 60 St. George St., Toronto, ON M5S 3H8, Canada \\ e-mail: almog.yalin@gmail.com \\ 2 Department of Physics, The George Washington University, Washington, DC 20052, USA \\ ${ }^{3}$ Astronomy, Physics and Statistics Institute of Sciences (APSIS), Washington, DC 20052, USA
}

Received 30 November 2017 / Accepted 20 March 2018

\section{ABSTRACT}

\begin{abstract}
Context. A previous study claimed the discovery of an intermediate-mass black hole (IMBH). This hypothetical black hole was invoked in order to explain the high-velocity dispersion in one of several dense molecular clouds near the Galactic center. The same study considered the possibility that this cloud was due to a supernova explosion, but disqualified this scenario because no X-rays were detected.

Aims. We here check whether a supernova explosion could have produced that cloud, and whether this explanation is more likely than an IMBH. More specifically, we wish to determine whether a supernova inside a dense molecular cloud would emit in the X-rays. Methods. We have approached this problem from two different directions. First, we performed an analytic calculation to determine the cooling rate by thermal bremsstrahlung and compared this time to the lifetime of the cloud. Second, we estimated the creation rate of these dense clouds in the central molecular zone (CMZ) region near the Galactic center, where they were observed. Based on this rate, we can place lower bounds on the total mass of IMBHs and clouds and compare this to the masses of the components of the CMZ. Results. We find that the cooling time of the supernova remnant inside a molecular cloud is shorter than its dynamical time. This means that the temperature in such a remnant would be much lower than that of a typical supernova remnant. At such a low temperature, the remnant is not expected to emit in the X-rays. We also find that to explain the rate at which such dense clouds are created requires fine-tuning the number of IMBHs.

Conclusions. We find the supernova model to be a more likely explanation for the formation of high-velocity compact clouds than an IMBH.
\end{abstract}

Key words. stars: black holes - ISM: clouds - supernovae: general - ISM: supernova remnants

\section{Introduction}

Recent observations revealed a number of peculiar molecular clouds, also known as high-velocity compact clouds (HVCCs), in the Galactic bulge (Tokuyama et al. 2017; Oka et al. 1999). Their properties are summarized in Table 1. The typical projected size of these clouds is less than $10 \mathrm{pc}$, and their spread in radial velocities is a few tens of $\mathrm{km} \mathrm{s}^{-1}$, significantly larger than expected from their motion in the Galaxy or from their selfgravity. The virial mass of each cloud is much greater than its actual mass, implying that these clouds are not bound by selfgravity. One particular cloud, $\mathrm{CO} 0.40-0.22$, rose to prominence as evidence for an intermediate-mass black hole (IMBH; Oka et al. 2015). It has been shown that the cloud's velocity dispersion might result from a scattering event by a compact object with a mass of $10^{5} M_{\odot}($ Oka et al. 2017).

Alternative explanations for the observed features have also been considered. One candidate is a supernova (Oka et al. 2017). While the kinetic energy of CO $0.40-0.22,10^{49.7} \mathrm{erg}$, is similar to that of a typical supernova (as are the energies of the other clouds; see Table 1), this scenario has been disqualified because no counterpart in other wavelengths has been observed, whereas a typical supernova remnant is expected to emit in the X-ray range (Cioffi et al. 1988).

Without strong evidence to the contrary, it is reasonable to assume that the observed HVCCs share a common origin. There are a few HVCCs, with typical lifetimes (inferred from their sizes and velocities) of $10^{5} \mathrm{yr}$, which suggests a formation rate of such clouds of at least $10^{-5} \mathrm{yr}^{-1}$. This alone implies that the origin of these clouds should be a relatively common one, and casts doubt on the IMBH scenario.
In this paper, we re-examine the supernova scenario. The problem of a supernova in a molecular cloud has been considered in the past by several authors (Körtgen et al. 2016; Walch \& Naab 2015; Chevalier 1974). These works considered a density higher than that of typical interstellar matter, but still below the threshold necessary to be dominated by radiation. We here consider a supernova shock wave propagating into a medium with an even higher density, where photon production drastically reduces the temperature. In turn, this reduction in temperature suppresses the emission of X-rays.

The paper is organized as follows. In Sect. 2 we explain the absence of the expected thermal emission from a supernova embedded in a molecular cloud. In Sect. 3 we present possible issues with the association of IMBHs with dense molecular clouds with high velocity spreads. In Sect. 4 we summarize and discuss some general implications of these results.

\section{Supernova scenario}

A typical supernova has energy around $10^{51}$ ergs (Kasen \& Woosley 2009). This is enough energy to endow CO 0.40-0.22 with its current kinetic energy $10^{49.7} \mathrm{erg}$. However, the supernova scenario has previously been disqualified since it was expected that a supernova remnant would emit copiously in the X-ray, and no such emission has been detected (Oka et al. 2017). In this section we show that in fact one should not expect X-rays even if the cloud's observed velocity dispersion is due to a previous supernova explosion. This is because the timescale over which the hot gas radiates its energy is shorter than the dynamical time of the system. As a conservative limit, we consider cooling due to thermal bremsstrahlung. We show that this mechanism alone 
Table 1. Parameters of the high-velocity compact molecular clouds.

\begin{tabular}{lcccccccc}
\hline \hline Designation & $r[\mathrm{pc}]$ & $\Delta V\left[\mathrm{~km} \mathrm{~s}^{-1}\right]$ & $M\left[M_{\odot}\right]$ & $M_{\mathrm{vir}}\left[M_{\odot}\right]$ & $E_{k}[\mathrm{erg}]$ & $t_{\mathrm{age}}[\mathrm{yr}]$ & $\rho\left[\mathrm{g} \mathrm{cm}^{-3}\right]$ & $\mathrm{Ref}$. \\
\hline CO 0.02-0.02 & 3.5 & 100 & $9 \times 10^{4}$ & $8.1 \times 10^{6}$ & $1.3 \times 10^{51}$ & $7 \times 10^{4}$ & $1.43 \times 10^{-19}$ & 1 \\
CO 1.28+0.06 & 8 & 100 & $\approx 4 \times 10^{4}$ & $1.9 \times 10^{7}$ & $6 \times 10^{50}$ & $1.6 \times 10^{5}$ & $5.31 \times 10^{-21}$ & 2 \\
CO 2.88+0.08 & 5 & 80 & $\approx 10^{4}$ & $7.4 \times 10^{6}$ & $10^{50}$ & $1.2 \times 10^{5}$ & $5.44 \times 10^{-21}$ & 2 \\
CO 3.34+0.43 & 8 & 30 & $\approx 4 \times 10^{4}$ & $1.7 \times 10^{6}$ & $5 \times 10^{49}$ & $5.3 \times 10^{5}$ & $5.31 \times 10^{-21}$ & 2 \\
CO 0.40-0.22 & 5 & 100 & 4000 & $1.2 \times 10^{7}$ & $6 \times 10^{49}$ & $10^{5}$ & $2.18 \times 10^{-21}$ & 3 \\
HCN 0.009-0.044 & 0.37 & 20 & 16 & $3.4 \times 10^{4}$ & $10^{46}$ & $3.7 \times 10^{4}$ & $2.51 \times 10^{-20}$ & 4 \\
HCN 0.085-0.094 & 0.33 & 40 & 13 & $1.2 \times 10^{5}$ & $3 \times 10^{46}$ & $1.6 \times 10^{4}$ & $2.46 \times 10^{-20}$ & 4 \\
\hline
\end{tabular}

Notes. Approximate sign in the mass column indicates that a line-based mass estimate was not available. In these cases, mass was estimated by multiplying the volume of the cloud with the average density of the line-emitting gas $\left(10^{4.5} \mathrm{~cm}^{-3}\right)$ times a reasonable filling factor $0.1 . r$ is the size of the cloud, $\Delta V$ is the velocity dispersion, and $M$ is the mass. The virial mass was calculated using the relation $M_{\mathrm{vir}}=G \Delta V^{2} \times r$. The kinetic energy was calculated using the relation $E_{k}=\frac{3}{10} M \Delta V^{2}$. The age was calculated using $t_{\text {age }}=r / \Delta v$. The references are (1) Oka et al. (1999), (2) Tokuyama et al. (2017), (3) Oka et al. (2015), and (4) Takekawa et al. (2017).

is sufficient to cool the gas over a timescale considerably shorter than the dynamical one.

The emissivity of thermal bremsstrahlung is given by (Rybicki \& Lightman 1986)

$\varepsilon_{b s} \approx \alpha \frac{m_{\mathrm{e}} c^{3}}{r_{\mathrm{e}}^{4}}\left(r_{\mathrm{e}}^{3} \frac{\rho}{m_{\mathrm{p}}}\right)^{2} \sqrt{\frac{k_{\mathrm{B}} T}{m_{\mathrm{e}} c^{2}}}$,

where $\alpha$ is the fine-structure constant, $m_{\mathrm{e}}$ is the mass of the electron, $c$ is the speed of light, $r_{\mathrm{e}}$ is the classical electron radius, $\rho$ is the mass density of the gas, $m_{\mathrm{p}}$ is the mass of the proton, $k_{\mathrm{B}}$ is the Boltzmann constant, and $T$ is the temperature. The thermal energy density of an ideal gas is $\rho k_{\mathrm{B}} T / m_{\mathrm{e}}$, and the ratio between the energy density and the emissivity gives the cooling time,

$t_{c} \approx \frac{m_{\mathrm{p}} \sqrt{k_{\mathrm{B}} T}}{\alpha c^{2} \sqrt{m_{\mathrm{e}}} \rho r_{\mathrm{e}}^{2}}$.

Equation (2) gives the timescale over which a gas radiates most of its energy if the only emission mechanism involved is thermal bremsstrahlung. The cooling time is an increasing function of temperature, so that until it reaches some terminal temperature (which we discussed below), the gas spends most of the time close to the initial temperature, which therefore dominates its overall cooling time.

A shock wave moving through a gas at velocity $v$, assuming no photons are produced, will heat up particles to a temperature

$k_{\mathrm{B}} T \approx m_{\mathrm{p}} v^{2}$.

Photon production by thermal bremsstrahlung will reduce the temperature, simply because the same amount of energy is divided among more particles. We calculate the (maximum) time it takes a gas to radiate its energy by plugging Eq. (3) into Eq. (2), along with appropriate values for CO 0.02-0.02

$t_{\mathrm{c}} \approx 10^{4} \frac{v}{50 \mathrm{~km} \mathrm{~s}^{-1}}\left(\frac{M_{\mathrm{c}}}{4000 M_{\odot}}\right)^{-1}\left(\frac{R}{5 \mathrm{pc}}\right)^{3}$ year,

where $M_{\mathrm{c}}$ is the mass of the cloud and $R$ is its size. This cooling time is shorter than the dynamical time by an order of magnitude. Even if photons do not leak out, photon production will continue, and the number of photons will increase. In this case, the number of photons will only stop increasing once a Planck equilibrium is reached. The temperature in a Planck equilibrium is given by $a T^{4} \approx \rho v^{2}$, where $a$ is the radiation constant. For CO $0.02-0.02$, the temperature in a Planck equilibrium is

$T \approx 1000\left(\frac{M_{\mathrm{c}}}{4000 M_{\odot}}\right)^{1 / 4}\left(\frac{R}{5 \mathrm{pc}}\right)^{-3 / 4}\left(\frac{v}{50 \mathrm{~s}^{-1}}\right)^{1 / 2} \mathrm{~K}$.

Gas at such a low temperature does not emit X-rays. The actual temperature will probably be even lower than that because of photon leakage and adiabatic cooling. If photons do leak out, the temperature will drop below the value in Eq. (5).

We note that this study only takes into account thermal bremsstrahlung. This is indeed the dominant emission mechanism for temperatures in excess of about $10^{7} \mathrm{~K}$ (Sutherland \& Dopita 1993). Below $10^{7} \mathrm{~K}$, other radiative processes kick in and further accelerate photon production. A cold gas swept by a shock wave moving at $100 \mathrm{~km} \mathrm{~s}^{-1}$ will initially heat up to $10^{6} \mathrm{~K}$. At these temperatures, the gas should be completely ionized, and the gas cools efficiently. However, below $10^{5} \mathrm{~K}$, cooling by recombination exceeds bremsstrahlung (Burrows \& Ostriker 2014), and when the temperature drops below about $10^{4} \mathrm{~K}$, the gas recombines. Recombination can suppress the thermal bremsstrahlung emission, but even if the gas does not cool considerably beyond this point, it will no longer emit in X-rays.

These calculations were done for the parameters of the cloud in its current state. This is a conservative assumption. In the past, the cloud must have been more compact. The dynamical time scales as $R$, while the cooling time from thermal bremsstrahlung scales as $R^{3}$ (Eq. (4)). Therefore, even though the dynamical time was shorter at earlier times, the cooling time was reduced by an even greater amount.

One possible issue with the supernova scenario is that the shock can destroy molecules and dust grains. These molecules are expected to re-form after the transition of the shock front, but this process can take a long time, possibly longer than the lifetime of the system. This difficulty could be averted if the shock develops a precursor wave due to photons leaking to the upstream material (Draine \& McKee 1993). These precursors can drastically decrease the peak temperature attained as a fluid element traverses the shock front and travels downstream. In addition, a shock wave moving at the current velocity dispersion of the cloud will only destroy half of the dust grains (Slavin et al. 2004). The surviving grains can serve as catalysts for molecule formation (Vidali 2004).

The supernova scenario is also supported by a statistical argument. A cloud formation rate of $10^{-4} \mathrm{yr}^{-1}$ is consistent with 
a supernova origin, as these have a much higher rate of $10^{-2} \mathrm{yr}^{-1}$ in the Galaxy (Diehl et al. 2006). Furthermore, massive starforming regions and hence the supernovae that follow them are known to preferentially occur within such dense molecular clouds (Lada \& Lada 2003; Saito et al. 2007; Heyer et al. 2016). Additional support to the supernova origin is given by expanding shell-like structures that are observed in some of the HVCCs, such as CO 1.28+0.06 (Oka et al. 2001) and CO 0.02-0.02 (Oka et al. 1999). These structures are thought to be cavities blown by multiple supernovae within the cloud. Although CO 0.40-0.22 does not seem to have a simple expanding shell structure, it has been shown to partially overlap with a large shell-like structure (Oka et al. 2015), suggesting indeed that one or more supernovae have occurred in the vicinity of the cloud. The exact physical conditions required for supernovae to create inside cavities (number of supernovae, density of the cloud, etc.) have not yet been explored in detail. This question should be further investigated with numerical simulations.

\section{Implications of intermediate-mass black holes}

So far, about four such clouds have been detected at distances of a few hundred parsecs from the Galactic center (Oka et al. 1998), one at a distance of $60 \mathrm{pc}$ (Oka et al. 2015), and two within $20 \mathrm{pc}$ (Takekawa et al. 2017; see Table 1). If even a few of these clouds are due to an IMBH and assuming for the moment that each cloud corresponds to a unique and different black hole, we can obtain a lower limit on the number of such black holes. The lifetime of these clouds is on the order of a hundred thousand years, after which they break up completely. Assuming a constant formation rate, this corresponds to a rate of at least $r_{\mathrm{c}} \approx 10^{-5} \mathrm{yr}^{-1}$. Thus, over the $\sim 10^{10} \mathrm{yr}$ lifetime of the Milky Way, roughly $10^{6}$ such clouds have been formed and the total mass of the black holes within $300 \mathrm{pc}$ of the Galactic center would be huge, about $10^{11} M_{\odot}$, which is larger than that of the Galactic bulge.

However, each IMBH could, in principle, produce multiple high-velocity clouds. This will of course reduce the naive requirement on the total mass in IMBHs presented above. Nonetheless, this will require a large number of such clouds, and hence a high mass in these clouds, as we show below. The rate of random isotropic collisions between compact clouds and IMBHs is given by

$r_{\mathrm{c}}=n_{\mathrm{cl}} v_{\mathrm{cl}} N_{\mathrm{H}} b^{2}$

where $n_{\mathrm{cl}}$ is the number density of clouds, $N_{h}$ is the number of black holes, $v_{\mathrm{cl}}$ is the velocity of the clouds prior to their collision with an IMBH (which is assumed to be given by the Galactic velocity dispersion at the same radius) and $b$ is the critical impact parameter between a cloud and a black hole below which the cloud will be disrupted. When we use Eq. (6), the net mass of the high-velocity clouds needed can be estimated

$$
\begin{aligned}
M_{\mathrm{c}} \approx & \frac{4 m_{\mathrm{c}} r_{\mathrm{o}}^{3} r_{\mathrm{c}}}{3 N_{\mathrm{h}} b^{2} v_{\mathrm{cl}}} \approx 5.2 \times 10^{7}\left(\frac{m_{\mathrm{c}}}{4000 M_{\odot}}\right)\left(\frac{r_{o}}{100 \mathrm{pc}}\right)^{3} \\
& \times\left(\frac{N_{\mathrm{h}}}{10^{2}}\right)^{-1}\left(\frac{b}{1 \mathrm{pc}}\right)^{-2}\left(\frac{r_{\mathrm{c}}}{10^{-5} \mathrm{yr} \mathrm{s}^{-1}}\right)\left(\frac{v_{\mathrm{cl}}}{10 \mathrm{~km} \mathrm{~s}^{-1}}\right)^{-1} M_{\odot}
\end{aligned}
$$

where $m_{\mathrm{c}}$ is the average mass of a high-velocity cloud and $r_{\mathrm{o}}$ is the Galactocentric radius to which they were observed. The total mass of these clouds is inversely proportional to the number of black holes. The mass of HVCCs cannot exceed the total mass of molecular gas in the central molecular zone (CMZ), around $4 \times 10^{7} M_{\odot}$ (Mills 2017). At the same time, the mass of the

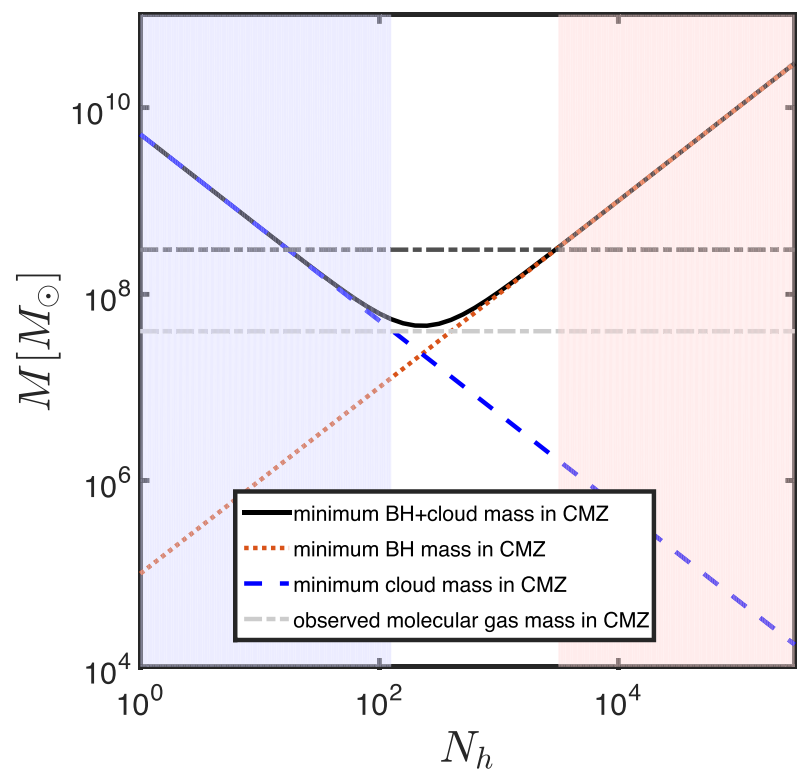

Fig. 1. Total implied mass in IMBHs and in HVCCs within $100 \mathrm{pc}$ of the Galactic center, assuming that all high-velocity clouds are due to interactions with such black holes. The blue shaded area represents the region of parameter space where the total implied mass of HVCCs exceeds the observed mass of the molecular gas. The red shaded area represents the region where the mass of IMBHs exceeds the mass of dark matter within $100 \mathrm{pc}$.

IMBHs cannot exceed the mass of dark matter. The total mass within $100 \mathrm{pc}$ is about $10^{9} M_{\odot}$ (Sofue 2013). Dark matter in the $\mathrm{CMZ}$ is less abundant than in the rest of the Galaxy, comprising only between $10 \%$ and $30 \%$ of the mass (Hooper 2017). Adopting a conservative estimate of $30 \%$ yields a dark matter mass within $100 \mathrm{pc}$ of about $3 \times 10^{8} M_{\odot}$. These constraints are summarized in Fig. 1. As can be seen in this figure, there is only a relatively narrow region $5 \times 10^{3}>N_{\mathrm{h}}>2 \times 10^{2}$ that does not violate the conditions discussed above. We note that $N_{\mathrm{h}}$ within these limits may still be problematic as it would require either that HVCC comprise a considerable fraction of the molecular gas, or that IMBHs comprise a considerable fraction of the dark matter mass.

One potential concern with this estimate is the prospect that an IMBH might interact with more tenuous clouds, which are more common in the CMZ, to produce hypervelocity compact clouds. This scenario, however, cannot account for the observed velocity dispersions of the clouds, as we demonstrate below. To understand the problem with this possibility, we consider the outcome of a gravitational scattering event between a stationary light particle and a particle with mass $M_{\mathrm{h}}$, moving at velocity $v_{\mathrm{cl}}$ and with impact parameter $b$. This problem has two velocity scales: the collision velocity $v_{\mathrm{cl}}$, and the virial velocity $v_{\mathrm{v}} \approx\left(G M_{h} / b\right)^{1 / 2}$. When $v_{\mathrm{cl}} \gg v_{\mathrm{v}}$, the asymptotic velocity difference of the light particle is $v_{\mathrm{v}}^{2} / v_{\mathrm{cl}} \leq v_{\mathrm{v}}$. In the opposite limit, $v_{\mathrm{cl}} \ll v_{\mathrm{v}}$, the asymptotic velocity difference is $2 v_{\mathrm{cl}}$. Therefore, the maximum asymptotic velocity that the particle can have after a scattering event is $\sim \min \left(v_{\mathrm{v}}, v_{\mathrm{cl}}\right)$. Typical clouds in the $\mathrm{CMZ}$ have an average density of about $10^{3} \mathrm{~cm}^{-3}$, so that a cloud of $4000 M_{\odot}$ would have a radius of about $4 \mathrm{pc}$ (see also Table 1), which for a typical particle in the cloud translates into a virial velocity of $10 \mathrm{~km} \mathrm{~s}^{-1}$. This is an order of magnitude lower than the velocity dispersion of CO 0.04-0.22. We conclude that interactions of black holes with these large clouds cannot effectively accelerate a significant portion of the clouds' particles to the observed velocities. 
Another argument limiting the number of IMBHs is the rate of tidal disruption events. A black hole will tidally disrupt stars within their loss cone (Rees 1988). The cross section for such an event is given by

$\sigma_{\mathrm{lc}} \approx \frac{G M_{\mathrm{h}}}{v_{\mathrm{v}}^{2}} R_{s}\left(\frac{M_{\mathrm{h}}}{m_{\mathrm{s}}}\right)^{1 / 3}$,

where $G$ is the universal constant of gravity, $M_{\mathrm{h}}$ is the mass of the IMBH, $R_{\mathrm{S}}$ is the stellar radius, $v_{\mathrm{v}}$ is the virial velocity of the HVCCs, and $m_{s}$ is the mass of the star. The timescale for tidal disruption of stars by a single IMBH is

$$
\begin{aligned}
t_{\mathrm{lc}} \approx & 2 \times 10^{9}\left(\frac{n_{\mathrm{s}}}{10^{2} \mathrm{pc}^{-3}}\right)^{-1} \frac{v_{\mathrm{v}}}{10^{2} \mathrm{~km} \mathrm{~s}^{-1}} \\
& \times\left(\frac{M_{\mathrm{h}}}{10^{5} M_{\odot}}\right)^{-4 / 3}\left(\frac{R_{\mathrm{s}}}{R_{\odot}}\right)^{-1}\left(\frac{m_{\mathrm{s}}}{M_{\odot}}\right)^{1 / 3} \mathrm{yr},
\end{aligned}
$$

where $n_{\mathrm{s}}$ is the number density of stars. When the number of IMBHs exceeds $10^{5}$, then the rate of tidal disruption events will exceed the theoretical rate expected due to the supermassive black hole at the Galactic center (once per $10^{4}$ years). The rate inferred from observations is even lower (Stone \& Metzger 2015), which sets an even more stringent upper limit on the number of IMBHs.

\section{Conclusions}

We have discussed the recent suggestion that a compact cloud with high-velocity spread serves as evidence for an IMBH. We proposed a less exotic scenario, where the same observational features can be reproduced by a supernova. A supernova has enough energy to endow the molecular cloud with its current velocity spread. Since such a supernova exploded in a very dense environment, photon production is much faster than in a typical supernova remnant. As photons are generated, the available energy is divided between more particles, and the temperature drops. We showed that in contrast to a typical supernova, material shocked by a supernova in a dense environment can cool below X-ray emitting temperatures on a timescale shorter than the dynamical time. We demonstrated this for thermal bremsstrahlung. Other emission mechanisms such as atomic and molecular lines can accelerate this process even further.

While our model agrees well with the observations and is statistically justifiable, we do not rule out the possibility that different mechanisms could form these clouds. One option that has recently been proposed is the collision of two clouds (Ravi et al. 2017). In order to distinguish between the two models, more observations are needed to catch these clouds at a younger age. If about ten clouds are observed with an age of around $10^{4.5} \mathrm{yr}$, then there is a reasonable chance for a cloud with an age of $10^{3.5} \mathrm{yr}$. At this age, in the supernova model proposed here, the cloud might be emitting in the infra-red or optical. In addition to observations, the study of these objects can benefit from hydrodynamic simulations with non-equilibrium radiation transfer.

In addition, we presented two arguments against the association of these clouds with black holes. Owing to the short lifetimes of these clouds, they must be produced at a rapid rate, which requires either very many seed clouds or IMBHs. To stay within the limits of the molecular gas and dark matter masses, the number of IMBHs cannot be less than $10^{2}$ or more than a few $10^{3}$. We note that a number of IMBHs within these limits may still be problematic as it would imply that either HVCCs constitute a considerable portion of the molecular gas mass, or that IMBHs constitute a considerable portion of the dark matter mass. The second argument is that these black holes may cause too many tidal disruption events, in excess of the rate inferred from observations. This problem is further exacerbated because IMBHs can disrupt compact objects such as white dwarfs, which is not the case for more massive black holes (Rosswog et al. 2009). While these arguments do not rule out the IMBH scenario, they make it unlikely. In other words, even if this mechanism does occur, it can only account for a small fraction of the HVCC.

Another feature associated with CO $0.40-0.22$ is a continuum millimeter wavelength source (Oka et al. 2015). The spectrum is sampled at two frequencies, 231 and $266 \mathrm{GHz}$, with fluxes 8.4 and $9.9 \mathrm{mJy}$, respectively. This emission is thought to be blackbody radiation from an object with a temperature of $9 \mathrm{~K}$. We can conceive of two possible explanations for this emission, other than an IMBH, as was suggested by Oka et al. (2015). One option is a protostar, which can reach a similar luminosity and temperature (Larson 1969). Another option is a neutron star. Other neutron stars in the Galactic center produce similar fluxes (Schnitzeler et al. 2016). A neutron star's flux in this frequency typically decreases with frequency, which is inconsistent with the observations. This inconsistency can be due to fluctuations that exceed the error margin, as can be seen in the observations of the Crab pulsar spectrum (Arendt et al. 2011). An examination of archival data favors a protostellar disk (Ravi et al. 2017), but more observations are required to shed light on the nature of the continuum source.

Acknowledgements. AY would like to thank Chris Matzner, Chris Thompson, and Patrick Breysse for useful discussions. PB would like to thank Joe Silk for helpful suggestions and comments. We would also like to thank the anonymous referee for the helpful comments.

\section{References}

Arendt, R. G., George, J. V., Staguhn, J. G., et al. 2011, ApJ, 734, 54 Burrows, A. S., \& Ostriker, J. P. 2014, Proc. Natl. Acad. Sci., 111, 2409 Chevalier, R. A. 1974, ApJ, 188, 501

Cioffi, D. F., McKee, C. F., \& Bertschinger, E. 1988, ApJ, 334, 252 Diehl, R., Halloin, H., Kretschmer, K., et al. 2006, Nature, 439, 45

Draine, B. T., \& McKee, C. F. 1993, Annu. Rev. Astron. Astrophys., 31, 373

Heyer, M., Gutermuth, R., Urquhart, J. S., et al. 2016, A\&A, 588, A29

Hooper, D. 2017, Phys. Dark Univ., 15, 53

Kasen, D., \& Woosley, S. E. 2009, ApJ, 703, 2205

Körtgen, B., Seifried, D., Banerjee, R., Vázquez-Semadeni, E., \& Zamora-Avilés, M. 2016, MNRAS, 459, 3460

Lada, C. J., \& Lada, E. A. 2003, Annu. Rev. Astron. Astrophys., 41, 57 Larson, R. B. 1969, MNRAS, 145, 271

Mills, E. A. C. 2017, ArXiv e-prints [arXiv: 1705.05332]

Oka, T., Hasegawa, T., Sato, F., Tsuboi, M., \& Miyazaki, A. 1998, ApJS, 118, 455 Oka, T., White, G. J., Hasegawa, T., et al. 1999, ApJ, 515, 249

Oka, T., Hasegawa, T., Sato, F., Tsuboi, M., \& Miyazaki, A. 2001, PASJ, 53, 779 Oka, T., Mizuno, R., Miura, K., \& Takekawa, S. 2015, ApJ, 816, L7

Oka, T., Tsujimoto, S., Iwata, Y., Nomura, M., \& Takekawa, S. 2017, Nat. Astron., 1, 709

Ravi, V., Vedantham, H., \& Phinney, E. S. 2017, MNRAS, submitted, [arXiv: 1710.03813 ]

Rees, M. J. 1988, Nature, 333, 523

Rosswog, S., Ramirez-Ruiz, E., \& Hix, W. R. 2009, ApJ, 695, 404

Rybicki, G. B., \& Lightman, A. P. 1986, Radiative Processes in Astrophysics (Weinheim: Wiley-VCH)

Saito, H., Saito, M., Sunada, K., \& Yonekura, Y. 2007, ApJ, 659, 459

Schnitzeler, D. H. F. M., Eatough, R. P., Ferrière, K., et al. 2016, MNRAS, 459, 3005

Slavin, J. D., Jones, A. P., \& Tielens, A. G. G. M. 2004, ApJ, 614, 796

Sofue, Y. 2013, PASJ, 65, 118

Stone, N. C., \& Metzger, B. D. 2015, MNRAS, 455, 859

Sutherland, R. S., \& Dopita, M. A. 1993, ApJS, 88, 253

Takekawa, S., Oka, T., Iwata, Y., Tokuyama, S., \& Nomura, M. 2017, ApJ, 843, L11

Tokuyama, S., Oka, T., Takekawa, S., et al. 2017, Proc. Int. Astron. Union, 11, 154

Vidali, G. 2004, J. Geophys. Res., 109, E07S14

Walch, S., \& Naab, T. 2015, MNRAS, 451, 2757 